\title{
Utilidad de la prueba de la nitrato reductasa para la detección rápida de resistencia en Mycobacterium tuberculosis
}

\author{
Lorena González¹, Ricardo Sánchez², Martha Isabel Murcia ${ }^{1}$ \\ Departamento de Microbiología, Facultad de Medicina, Universidad Nacional de Colombia, Bogotá, D.C., Colombia \\ 2 Departamento de Psiquiatría, Facultad de Medicina, Universidad Nacional de Colombia, Bogotá, D.C., Colombia
}

Introducción. La detección temprana de la resistencia en Mycobacterium tuberculosis es de gran importancia para el manejo del paciente y el control de la infección.

Objetivo. Evaluar el rendimiento de la prueba de la nitrato reductasa para la determinación de la resistencia de $M$. tuberculosis a los siguientes medicamentos antituberculosos de primera línea: rifampicina, isoniacida, estreptomicina y etambutol.

Materiales y métodos. Se comparó la utilidad de la prueba de la nitrato reductasa con el método de las proporciones múltiples para determinar la resistencia de aislamientos de $M$. tuberculosis.

Resultados. Comparada con la del método de las proporciones múltiples, la sensibilidad de la prueba de la nitrato reductasa para rifampicina, isoniacida, estreptomicina y etambutol fue de 92, 91, 63 y $80 \%$, respectivamente, en tanto que la especificidad fue de 100,100, 100 y $98 \%$, respectivamente. Los valores pronósticos positivos fueron, en su orden, 100, 100, 100 y $80 \%$ para rifampicina, isoniacida, estreptomicina y etambutol, y los valores pronósticos negativos fueron 97, 93, 73 y $98 \%$, respectivamente. El tiempo medio de obtención de resultados fue menor cuando se uso la prueba de la nitrato reductasa (10 días) comparado con el del método de proporciones múltiples (28 días). Se observó una excelente concordancia entre los dos métodos fenotípicos de 98, 96, 81 y $96 \%$ para rifampicina, isoniacida, estreptomicina y etambutol, respectivamente.

Conclusiones. Los resultados demostraron que la prueba de la nitrato reductasa es útil para la detección precoz de farmacorresistencia y una herramienta valiosa para la detección rápida de la susceptibilidad de M. tuberculosis a los fármacos antituberculosos en países con bajos recursos.

Palabras clave: tuberculosis, Mycobacterium tuberculosis, resistencia a medicamentos, nitratoreductasa, pruebas de sensibilidad microbiana.

doi: http://dx.doi.org/10.7705/biomedica.v34i0.1694

The usefulness of the nitrate reductase assay for detecting drug-resistant Mycobacterium tuberculosis

Introduction: The early detection of resistance in Mycobacterium tuberculosis is of primary importance for both patient management and infection control.

Objective: To evaluate nitrate reductase assay (NRA) performance for the testing of Mycobacterium tuberculosis drug-resistance against first-line anti-tuberculosis drugs, such as rifampicin (RIF), isoniazid (INH), streptomycin (STR) and ethambutol (EMB).

Materials and methods: Fifty isolates were tested by using both the proportion method and the nitrate reductase assay.

Results: RIF, INH, STR and EMB sensitivity was found to be $92 \%, 91 \%, 63 \%$ and $80 \%$ and $100 \%$, respectively, and a corresponding specificity of $100 \%, 100 \%, 100 \%$ and $98 \%$ by comparing NRA results to those obtained with the gold standard (i.e., the proportion method). The positive predictive values for RIF, INH, STR and EMB were $100 \%, 100 \%, 100 \%$ and $80 \%$ and the negative predictive values were $97 \%, 93 \%, 73 \%$ and $98 \%$, respectively. The mean time for obtaining results was shorter when using the nitrate reductase assay (10 days) compared to using the proportion method (28 days). Excellent agreement was observed between both phenotypic tests: $98 \%, 96 \%, 81 \%$ and $96 \%$ for RIF, INH, STR and EMB, respectively.

Conclusions: The results showed that the nitrate reductase assay is suitable for the early determination

\footnotetext{
Contribución de los autores:

Lorena González: revisión de la literatura científica, ejecución de la parte experimental, borrador del manuscrito.

Ricardo Sánchez: revisión de la literatura científica, análisis estadístico, redacción de la discusión y corrección del manuscrito.

Martha Murcia: supervisión de la parte experimental, análisis de resultados, revisión de la literatura científica, redacción, discusión y corrección del manuscrito.
} 
of multidrug-resistant tuberculosis (MDR-TB) and is a useful tool for the quick and accurate determination of a rapid $M$. tuberculosis drug-sensitivity test in countries having low resources.

Key words: Tuberculosis, Mycobacterium tuberculosis, drug resistance, nitrate-reductase, microbial sensitivity tests.

doi: http://dx.doi.org/10.7705/biomedica.v34i0.1694

La tuberculosis continúa siendo una amenaza grave para la salud pública mundial. Cada año se presentan más de nueve millones de casos nuevos en todo el mundo y la tasa de incidencia disminuye menos de $1 \%$ por año (1). El control mundial de la tuberculosis se enfrenta a grandes desafíos tales como la circulación de cepas de Mycobacterium tuberculosis monorresistentes (2), farmacorresistentes (2,3, Puerto G, Wintaco M, Llerena C, Guerrero MI. Genotipos de Mycobacterium tuberculosis, circulantes en el puerto de Buenaventura, Colombia. Biomédica. 2011;31(Supl.3):57. Memorias, XX Congreso Latinoamericano de Parasitología), extremadamente resistentes (XDR) (Orjuela D, Garzón MC, García LM, Llerena C, Zabaleta A, Bueno J. Sensibilidad de Mycobacterium tuberculosis a fármacos antituberculosos de segunda línea en Colombia. Biomédica. 2011;31(Supl.3):55. Memorias, XX Congreso Latinoamericano de Parasitología) y totalmente resistentes (XXDR) (4-9), así como a la coinfección con el $\mathrm{VIH} /$ sida, la cual sigue alimentando la epidemia de tuberculosis $(1,10), y$ la falta de acceso de muchas de las poblaciones vulnerables a servicios de salud de calidad, todo esto sumado a la deficiente disponibilidad de tecnologías diagnósticas y a la aplicación de tratamientos erráticos y medidas de prevención inadecuadas $(1,10)$.

El principal reto para el control de la enfermedad es el incremento de casos de tuberculosis resistente a múltiples fármacos (farmacorresistente), por lo que la Organización Mundial de la Salud (OMS) ha impulsado para el año 2015 el acceso universal al diagnóstico y el tratamiento de la tuberculosis farmacorresistente y extremedamente resistente (11).

El diagnóstico precoz de la tuberculosis farmacorresistente es esencial para administrar a los pacientes el tratamiento adecuado y disminuir

\footnotetext{
Correspondencia:

Martha Isabel Murcia, Departamento de Microbiología, Facultad de Medicina, Universidad Nacional de Colombia, Carrera $30 \mathrm{~N}^{\circ}$ 45-03, edificio 417, oficina 312, Bogotá, D.C., Colombia Tel. (051) 316 5476; fax: (051) 3165476

mimurciaa@unal.edu.co

Recibido: 05/06/13; aceptado: 08/08/13
}

así la transmisión de cepas resistentes en la comunidad. Los métodos disponibles son muy costosos o sus resultados tardan mucho tiempo, por lo que en los países de bajos recursos se debe disponer de métodos sencillos, poco costosos y confiables que en corto tiempo permitan determinar los perfiles de sensibilidad de las cepas de M. tuberculosis circulantes.

Existe una gran variedad de técnicas para la detección de la resistencia de $M$. tuberculosis a los fármacos antituberculosos, entre las cuales el método de proporciones múltiples en su versión simplificada (12) está catalogado como prueba de referencia $(13,14)$, pero tiene como desventaja el tiempo de obtención de resultados, que oscila entre cuatro y seis semanas, ya que se basa en el crecimiento bacteriano detectado con medicamento y sin él mediante la visualización de colonias en medio de Löwenstein-Jensen (15).

También existen métodos comerciales más rápidos

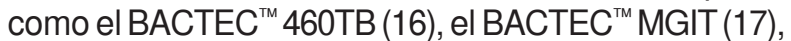
los cuales se basan en el crecimiento bacteriano en presencia del antibiótico, y los métodos genotípicos como el INNOLipa ${ }^{\text {TM }}$ _Rif.TB (Inmunogenetics) (18), el Geno Type ${ }^{\text {TM }}$ MTBDR (Hain Lifescience, Nehren, Germany) (19) y el Xpert ${ }^{\text {TM }}$ MTB/RIF (Cepheid) (20), que evalúan la resistencia por medio de la detección de mutaciones cromosómicas, y que, a pesar de ser rápidos, requieren personal entrenado, infraestructura adecuada, reactivos y equipos sofisticados y costosos, lo que hace difícil su uso en países de recursos limitados como el nuestro (21).

Por lo tanto, se necesitan métodos alternativos comparables con los métodos de referencia que permitan la obtención de resultados en el menor tiempo posible, que no representen riesgo de infección para el personal que los utiliza y que, además, no tengan costos elevados.

La prueba de la nitrato reductasa, inicialmente desarrollada por Golyshevskaia, et al., en 1996 (22), es un método colorimétrico que se basa en la reducción de nitratos a nitritos, característica utilizada como indicador de crecimiento, y que se evidencia por el cambio de color al añadir un revelador. Esta prueba ha mostrado buena 
sensibilidad y especificidad (>90 \%) (14) al compararla con la prueba de proporciones múltiples considerada como la de referencia $(13,14)$, y no requiere equipos ni reactivos costosos, por lo que ha sido propuesta como una herramienta fenotípica alternativa, útil para la detección rápida y precisa de la resistencia de $M$. tuberculosis a fármacos de primera línea $(13,14,23-27)$. Además, la utilización de medios sólidos (Löwenstein-Jensen y agar Middlebrook 7H11), a los que se les agregan el nitrato de potasio $\left(\mathrm{KNO}_{3}\right)$ como sustrato de la enzima y los medicamentos a evaluar, reduce el riesgo de producción de aerosoles, presente cuando se emplea un medio líquido (28).

Por las razones expuestas, el presente estudio evaluó el rendimiento de la prueba de la nitrato reductasa frente al método de las proporciones múltiples, considerado como prueba de referencia $(13,14)$, para la detección de la resistencia de aislamientos de $M$. tuberculosis a los fármacos antituberculosos de primera línea rifampicina, isoniacida, estreptomicina y etambutol.

\section{Materiales y métodos}

\section{Cepas}

Se utilizaron 50 cepas de M. tuberculosis conservadas en el Banco Biológico del Laboratorio de Micobacterias de la Facultad de Medicina de la Universidad Nacional de Colombia, con perfil de sensibilidad conocido para los medicamentos antituberculosos de primera línea determinado previamente por el método de proporciones múltiples. Como control se utilizó la cepa de $M$. tuberculosis $H 37 R v$, sensible a todos los fármacos por evaluar.

\section{Medicamentos antituberculosos}

Se utilizaron los siguientes fármacos en polvo de la marca Sigma Chemical Co. (St Louis, MO, USA): rifampicina (Sigma R7382), isoniacida (Sigma I-3377), estreptomicina (Sigma D-5155) y etambutol (Sigma E-4630) (29). Excepto la de la rifampicina, las soluciones madre de las drogas se prepararon con una concentración de 10.000 ug/ $\mathrm{ml}$ en agua destilada; la rifampicina se preparó con una concentración de $20.000 \mu \mathrm{g} / \mathrm{ml}$ en metanol. Las concentraciones finales fueron de $0,2 \mu \mathrm{g} / \mathrm{ml}$ de isoniacida, $4 \mu \mathrm{g} / \mathrm{ml}$ de estreptomicina, $2 \mu \mathrm{g} / \mathrm{ml}$ de etambutol y $40 \mu \mathrm{g} / \mathrm{ml}$ de rifampicina (29).

\section{Prueba de las proporciones múltiples}

Este método se utilizó siguiendo la descripción hecha por Canetti, et al. (30), con las concentraciones críticas recomendadas: rifampicina, $40 \mu \mathrm{g} /$ $\mathrm{ml}$; isoniacida, $0,2 \mu \mathrm{g} / \mathrm{ml}$; estreptomicina, $4 \mu \mathrm{g} / \mathrm{ml}$ y etambutol, $2 \mu \mathrm{g} / \mathrm{ml}$. Las pruebas se leyeron a los 28 días de incubación y, en caso de detectarse resistencia, no se hacían más lecturas. Cuando los resultados eran sensibles a los 28 días, se prolongaba la incubación y se hacía una lectura final a los 42 días. Los resultados de esta prueba se usaron como referencia para evaluar los resultados de la prueba de la nitrato reductasa.

\section{Prueba de la nitrato reductasa}

La realización de esta prueba se hizo de acuerdo al manual de procedimientos para la prueba del Instituto de Medicina Tropical de Bélgica elaborado por Martin y Palomino; como sustrato se utilizaron $1.000 \mu \mathrm{g} / \mathrm{ml}$ de nitrato de potasio $\left(\mathrm{KNO}_{3}\right)$ (29). Las concentraciones críticas de las drogas usadas fueron las mismas utilizadas en el método de proporciones múltiples.

Todas las cepas se repicaron en medio OgawaKudoh con el fin de obtener cultivos jóvenes de 21 a 28 días. A partir de los cultivos se preparó una suspensión homogénea a la que posteriormente se le ajustó la turbidez según el estándar de McFarland No. 1; después, se diluyó en proporción de 1/10 en agua destilada.

El montaje de la prueba de sensibilidad para cada cepa requería de tres tubos de control libres de medicamentos correspondientes a los 7, 10 y 14 días de incubación, días en que se hizo la lectura, y dos tubos por cada antibiótico evaluado. Los tubos de control se inocularon con $0,2 \mathrm{ml}$ de la dilución $1 / 10$ de la suspensión bacteriana ajustada al estándar y los tubos con antibióticos se inocularon con $0,2 \mathrm{ml}$ de la suspensión ajustada al McFarland No. 1 sin diluir.

Para revelar la prueba se hicieron tres lecturas en los días 7, 10 y 14 de incubación a $37^{\circ} \mathrm{C}$. Se procedió a leer el tubo de control correspondiente al día de lectura de cada cepa empleando la siguiente mezcla de reactivos como revelador: una parte de ácido clorhídrico, 1:1, dos partes de sulfanilamida, 0,2 \%, y dos partes de dihidrocloruro $\mathrm{N}$-naftiletilendiamina al $0,1 \%$; la mezcla se preparaba y se usaba inmediatamente. A cada tubo de control se le agregaron $0,5 \mathrm{ml}$ de esta mezcla; si aparecía algún color en el tubo de control que había sido revelado, se revelaban de la misma forma los tubos correspondientes que contenían los medicamentos; si este no era el caso, no se revelaba ningún tubo y se volvían a incubar (29). 


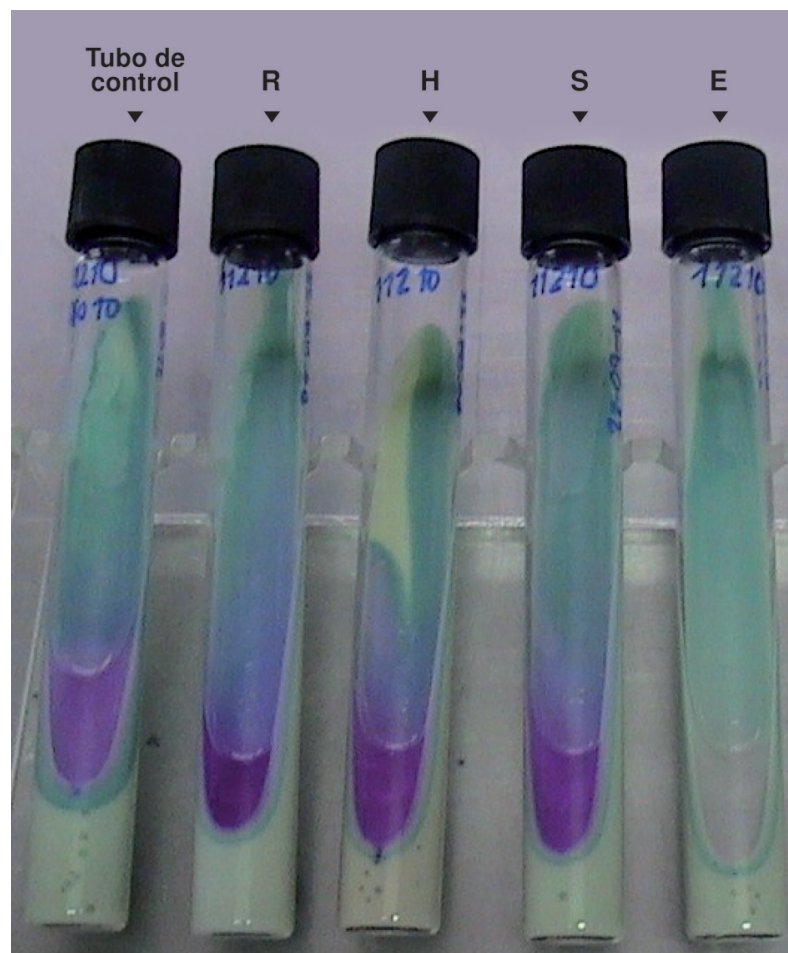

Figura 1. Prueba de la nitrato reductasa: rifampicina $(R)$; isoniacida $(H)$; estreptomicina $(S)$; etambutol $(E)$. Cepa resistente a rifampicina, isoniacida y estreptomicina, y sensible a etambutol. La mayor intensidad de color de los tubos con rifampicina, isoniacida y estreptomicina comparada con el color desarrollado en el tubo de control de crecimiento indica resistencia de la cepa al medicamento. La menor intensidad de color del tubo correspondiente a etambutol comparado con el de control indica sensibilidad.

En cuanto al perfil de sensibilidad de las cepas, estas se consideraban resistentes cuando el color de los tubos con antibiótico era mayor o igual al color desarrollado en el tubo de control revelado, y se las consideraba sensibles si no aparecía ningún color o era de menor intensidad que el color desarrollado en el tubo de control (29) (figura 1).

\section{Análisis de datos}

Los resultados de la prueba de la nitrato reductasa se analizaron tomando como referencia los valores arrojados por la prueba de proporciones múltiples (prueba de referencia). Para cada uno de los cuatro antibióticos utilizados se calcularon los valores de prevalencia de la resistencia, la sensibilidad, la especificidad, y los valores pronóstico, y, asimismo se establecieron los intervalos de confianza de $95 \%$. Los análisis se realizaron con el programa Stata $12^{\text {TM }}$ (Stata Corp., College Station, TX).

\section{Resultados}

Se evaluaron 50 aislamientos de $M$. tuberculosis con la prueba de la nitrato reductasa; 48 cepas dieron resultados sensibles de ser interpretados y dos aislamientos dieron resultado negativo.

Las sensibilidades a los fármacos de primera línea obtenidas con el método de la prueba de la nitrato reductasa comparado con el método de proporciones múltiples fueron las siguientes: 12 cepas resistentes, 35 sensibles y un falso sensible para rifampicina; 20 cepas resistentes, 26 sensibles y dos falsos sensibles para isoniacida; 15 cepas resistentes, 24 sensibles y nueve falsos sensibles para estreptomicina, y cuatro cepas resistentes, 42 sensibles, un falso sensible y un falso resistente para etambutol.

En el cuadro 1 se presentan los valores de sensibilidad y especificidad, así como los valores pronóstico positivos y negativos y los intervalos de confianza de $95 \%$ para cada medicamento estudiado.

Las sensibilidades más altas se obtuvieron para la rifampicina y la isoniacida (92 y $91 \%$, respectivamente), en tanto que la sensibilidad más baja se presentó en la estreptomicina (63\%). Las concordancias para rifampicina, isoniacida, estreptomicina y etambutol fueron de 93, 96, 81 y $96 \%$, respectivamente.

Los resultados de la prueba de la nitrato reductasa se obtuvieron en un intervalo de siete a 14 días; en $71 \%$ de los aislamientos los resultados se obtuvieron a los 10 días de incubación.

\section{Discusión}

La detección rápida de la farmacorresistencia es una prioridad para el control de la tuberculosis en el mundo. Los métodos comerciales que utilizan medio líquido y las pruebas moleculares de hibridación inversa en tira han sido aprobadas por la OMS como ensayos de referencia para la detección rápida de multirresistencia, sin embargo, debido a su complejidad técnica, su costo y los requerimientos de infraestructura, su uso es posible solo en regiones con grandes recursos (27). Dadas estas limitaciones, se han desarrollado métodos no comerciales de bajo costo como la prueba de la nitrato reductasa (27).

Con el fin de determinar las metas de eficiencia para el manejo y la evaluación de las pruebas de sensibilidad a los fármacos antituberculosos por parte del programa de garantía de la calidad (OMS/ 
Cuadro 1. Comparación de resultados de las pruebas de sensibilidad de 48 aislamientos de Mycobacterium tuberculosis con el método de proporciones múltiples y la prueba de la nitrato reductasa

\begin{tabular}{|c|c|c|c|c|c|c|c|}
\hline \multirow[t]{2}{*}{ Medicamento } & \multirow{2}{*}{$\begin{array}{l}\text { Proporciones } \\
\text { múltiples }\end{array}$} & \multicolumn{2}{|c|}{ Número } & \multicolumn{4}{|c|}{ Nitrato reductasa } \\
\hline & & Resistente & Sensible & $\begin{array}{c}\text { Sensibilidad } \\
(\%) \\
I C_{95 \%}\end{array}$ & $\begin{array}{c}\text { Especificidad } \\
(\%) \\
I_{95 \%}\end{array}$ & $\begin{array}{l}\text { VPP (\%) } \\
\text { IC }_{95 \%}\end{array}$ & $\begin{array}{l}\text { VPN (\%) } \\
\text { IC }_{95 \%}\end{array}$ \\
\hline \multirow[t]{2}{*}{ Rifampicina } & Resistente & 12 & 1 & 92 & 100 & 100 & 97 \\
\hline & Sensible & 0 & 35 & $64-99,8$ & $90-100$ & $73,5-100$ & $85,5-99,9$ \\
\hline \multirow[t]{2}{*}{ Isoniacida } & Resistente & 20 & 2 & 91 & 100 & 100 & 93 \\
\hline & Sensible & 0 & 26 & $70,8-98,9$ & $86,8-100$ & $83,2-100$ & $76,5-99,1$ \\
\hline \multirow[t]{2}{*}{ Estreptomicina } & Resistente & 15 & 9 & 63 & 100 & 100 & 73 \\
\hline & Sensible & 0 & 24 & $40,6-81,2$ & $85,8-100$ & $78,2-100$ & $54,5-86,7$ \\
\hline \multirow[t]{2}{*}{ Etambutol } & Resistente & 4 & 1 & 80 & 98 & 80 & 98 \\
\hline & Sensible & 1 & 42 & $28,4-99,5$ & $87-99,9$ & $28,4-99,5$ & $87,7-99,9$ \\
\hline
\end{tabular}

VPP: valor pronóstico positivo; VPN: valor pronóstico negativo

UICTER - Red Supranacional de Laboratorios de Referencia), Lazlo, et al., analizaron los resultados de las pruebas de sensibilidad en 100 cepas de M. tuberculosis realizadas por 14 laboratorios participantes y encontraron las sensibilidades más altas para rifampicina, $99 \%$, e isoniacida, $97 \%$, y las más bajas, para estreptomicina y etambutol con 93 y $90 \%$, respectivamente (31). Los autores determinaron los valores promedio de eficiencia para los diferentes fármacos así: para rifampicina, $99 \%$; para isoniacida, $97 \%$; para estreptomicina, $92 \%$, y para etambutol, $92 \%$. Los niveles de eficiencia menores de $95 \%$ en la rifampicina; de $89 \%$ en la isoniacida; de $80 \%$ en la estreptomicina, y de $89 \%$ en el etambutol indican un rendimiento bajo de las pruebas (31).

De acuerdo con estos valores, los resultados obtenidos en nuestro estudio, a excepción de la estreptomicina, mostraron una buena concordancia entre los dos métodos (rifampicina, $98 \%$, isoniacida, $96 \%$, estreptomicina, $81 \%$ y etambutol, $96 \%$ ); sin embargo, el nivel de eficiencia de la estreptomicina no fue menor a $80 \%$.

La sensibilidades más bajas encontradas en el presente estudio fueron la de la estreptomicina (63\%) y la del etambutol ( $80 \%)$, resultados similares a los hallazgos de López, et al., en Argentina, quienes al evaluar 302 aislamientos de M. tuberculosis colombianos empleando estas dos metodologías, encontraron sensibilidades a estreptomicina y etambutol de 59 y $55 \%$, respectivamente, y especificidades para los dos medicamentos de $99 \%$ (26), mientras que para rifampicina e isoniacida, las sensibilidades y especificidades halladas fueron de 93 y $84,9 \%$, respectivamente, con especificidades de $100 \%$ para los dos medicamentos (26).
En un estudio que evaluó en Nigeria 70 aislamientos de $M$. tuberculosis con los dos métodos, Any, et al., también reportaron resultados similares a los nuestros con sensibilidades y especificidades para estreptomicina y etambutol de 66,6 y $75 \%$, respectivamente, y especificidades de 91,8 y $100 \%$, comparados con las sensibilidades a rifampicina e isoniacida que fueron de 90 y $100 \%$, y especificidades de 96,6 y $100 \%$, respectivamente (32).

Como es bien sabido, la estreptomicina y el etambutol son fármacos difíciles de probar, incluso por medio de los métodos convencionales estándar (31).

Los resultados de nuestro estudio también son comparables a los reportados por el Instituto Nacional de Salud de Colombia (33), que evaluó la concordancia de la prueba de la nitrato reductasa indirecta con el método de proporciones múltiples en 183 cepas de $M$. tuberculosis y encontró valores de sensibilidad de $100 \%$ y de especificidad de $99 \%$ para la rifampicina y para la isoniacida de 86 y $94,7 \%$, respectivamente (33).

Varios estudios previos han encontrado discrepancias en la determinación de la sensibilidad a la estreptomicina: Angeby, et al. (22), reportaron sensibilidad de 95 y especificidad de $83 \%$; Shikama, et al. (34), lograron una concordancia de $90,8 \%$, y Martin, et al. (35), obtuvieron valores de sensibilidad de entre 41,7 y $91,6 \%$ y variaciones en la especificidad en la evaluación de la sensibilidad a estreptomicina en un estudio multicéntrico en el que participaron cinco laboratorios; los autores consideraron que estas discordancias podrían explicarse por la interacción aún desconocida del medicamento con el sustrato utilizado $\left(\mathrm{KNO}_{3}\right)$ (35). 
Un estudio reciente realizado en la India evaluó 286 aislamientos de $M$. tuberculosis con el método de la prueba de la nitrato reductasa y con el método de proporciones múltiples y encontró sensibilidades a los cuatro fármacos bajo estudio de 98,4, 97, 88,5 y $94,2 \%$, respectivamente, y especificidades de 100, 100, 94 y $99 \%$, que son similares a las encontradas en nuestro estudio (36). También son similares nuestros resultados a los obtenidos en Cuba en un estudio que analizó la sensibilidad de 320 cepas de $M$. tuberculosis a rifampicina, isoniacida, estreptomicina y etambutol y encontró una concordancia entre los dos métodos de 98,8\% (37).

Las ventajas del método de la prueba de la nitrato reductasa son la rapidez para visualizar el crecimiento, la simplicidad del procedimiento y el bajo costo, en tanto que las desventajas radican en la ausencia de la enzima nitrato reductasa en menos de $1 \%$ de las cepas de $M$. tuberculosis (38), por lo que tampoco es útil en Mycobacterium bovis, especie que es negativa para el nitrato. Además, debe tenerse en cuenta que algunas especies de micobacterias no tuberculosas como Mycobacterium kansasii, Mycobacterium szulgai, Mycobacterium flavescens, así como el complejo Mycobacterium terrae y algunas micobacterias de crecimiento rápido, producen la enzima nitrato reductasa $(36,38)$.

Es bien conocido, igualmente, que algunas bacterias reducen los nitratos a nitritos y posteriormente pueden pasar a productos más reducidos como el amoniaco, el nitrógeno molecular, el óxido nítrico, el óxido nitroso o la hidroxilamina, los cuales no pueden ser detectados por el revelador empleado, caso en que debe añadirse polvo de zinc para evidenciar esta última reacción $(21,36)$. En el presente estudio no se pudieron evaluar dos aislamientos por no haber desarrollado color; una de las causas pudo ser la formación de productos que no pueden ser detectados con el revelador empleado o debido a la ausencia de la enzima nitrato reductasa.

Los resultados obtenidos en este estudio confirman que la prueba de la nitrato reductasa es una herramienta útil, económica y rápida para la detección de perfiles de susceptibilidad de cepas de $M$. tuberculosis circulantes en países con bajos recursos.

\section{Agradecimientos}

Los autores agradecen al Laboratorio de Micobacterias del Instituto Nacional de Salud de Colombia por proporcionarnos el $\mathrm{KNO}_{3}$.

\section{Conflicto de intereses}

Los autores manifiestan que no existe conflicto de intereses.

\section{Financiación}

El presente proyecto fue financiado por el Departamento de Microbiología, Facultad de Medicina, Universidad Nacional de Colombia.

\section{Referencias}

1. Lonnroth K, Castro KG, Chakaya JM, Chauhan LS, Floyd K, Glaziou P, et al. Tuberculosis control and elimination 2010-50: Cure, care, and social development. Lancet. 2010;375:1814-29. http://dx.doi.org/10.1016/S01406736(10)60483-7

2. Garzón MC, Angee DY, Llerena C, Orjuela DL, Victoria JE. Vigilancia de la resistencia del Mycobacterium tuberculosis a los fármacos antituberculosos, Colombia 2004-2005. Biomédica. 2008;28:319-26.

3. Murcia MI, Manotas M, Jiménez YJ, Hernández J, Cortés MI, López LE, et al. First case of multidrug-resistant tuberculosis caused by a rare "Beijing-like" genotype of Mycobacterium tuberculosis in Bogotá, Colombia. Infect Genet Evol. 2010;10:678-81. http://dx.doi.org/10.1016/j.meegid. 2010.03.010

4. Udwadia ZF, Amale RA, Ajbani KK, Rodrigues C. Totally drug-resistant tuberculosis in India. Clin Infect Dis. 2012; 54:579-81. http://dx.doi.org/10.1093/cid/cir889

5. Mudur G. Indian health ministry challenges report of totally drug resistant tuberculosis. BMJ. 2012;344:e702. http://dx. doi.org/10.1136/bmj.e702

6. Loewenberg $\mathbf{S}$. India reports cases of totally drug-resistant tuberculosis. Lancet. 2012;379:205. http://dx.doi.org/10.1016/ S0140-6736(12)60085-3

7. Dahle UR. Totally drug resistant tuberculosis?. Tidsskr Nor Laegeforen. 2012;132:634. http://dx.doi.org/10.4045/tidsskr. 12.0168

8. Migliori GB, Centis R, D'Ambrosio L, Spanevello A, Borroni E, Cirillo DM, et al. Totally drug-resistant and extremely drug-resistant tuberculosis: The same disease? Clin Infect Dis. 2012;54:1379-80. http://dx.doi.org/10.1093/ $\mathrm{cid} / \mathrm{cis} 128$

9. Klopper M, Warren RM, Hayes C, Gey van Pittius NC, Streicher EM, Muller B, et al. Emergence and spread of extensively and totally drug-resistant tuberculosis, South Africa. Emerg Infect Dis. 2013;19:449-55. http://dx.doi.org/ 10.3201//EID1903.120246

10. Small PM. Tuberculosis: A new vision for the 21st century. Kekkaku. 2009;84:721-726.

11. World Health Organization. Towards universal access to diagnosis and treatment of multidrug-resistant and extensively drug-resistant tuberculosis by 2015: WHO progress report 2011. Geneva: World Health Organization; 2011.

12. Canetti G, Rist N, Grosset J. Measurement of sensitivity of the tuberculous bacillus to antibacillary drugs by the method of proportions. Methodology, resistance criteria, results and interpretation. Rev Tuberc Pneumol (Paris). 1963;27:217-72. 
13. Montoro E, Lemus D, Echemendia M, Martin A, Portaels F, Palomino JC. Comparative evaluation of the nitrate reduction assay, the MTT test, and the resazurin microtitre assay for drug susceptibility testing of clinical isolates of Mycobacterium tuberculosis. J Antimicrob Chemother. 2005; 55:500-5. http://dx.doi.org/10.1093/jac/dki023

14. Mengatto L, Chiani Y, Imaz MS. Evaluation of rapid alternative methods for drug susceptibility testing in clinical isolates of Mycobacterium tuberculosis. Mem Inst Oswaldo Cruz. 2006;101:535-42. http://dx.doi.org/10.1590/S0074-0276 2006000500009

15. Parsons LM, Somoskovi A, Urbanczik R, Salfinger $\mathbf{M}$. Laboratory diagnostic aspects of drug resistant tuberculosis. Front Biosci. 2004;9:2086-105. http://dx.doi.org/10.2741/1290

16. Mishra B, Rockey SM, Gupta S, Srinivasa H, Muralidharan S. Multi-drug-resistant tuberculosis: The experience of an urban tertiary care hospital in South India using automated BACTEC 460 TB. Trop Doct. 2012;42:35-37. http://dx.doi. org/110.1258/td.2011.110247

17. Said HM, Kock MM, Ismail NA, Baba K, Omar SV, Osman AG, et al. Comparison between the BACTEC MGIT 960 system and the agar proportion method for susceptibility testing of multidrug resistant tuberculosis strains in a high burden setting of South Africa. BMC Infect Dis. 2012;12:369. http://dx.doi.org/10.1186/1471-2334-12-369

18. Skenders GK, Holtz TH, Riekstina V, Leimane V. Implementation of the INNO-LiPA Rif. TB(R) line-probe assay in rapid detection of multidrug-resistant tuberculosis in Latvia. Int J Tuberc Lung Dis. 2011;15:1546-52. http:// dx.doi.org/10.5588/ijtld.11.0067

19. Barnard M, Gey van Pittius NC, van Helden PD, Bosman M, Coetzee G, Warren RM. The diagnostic performance of the GenoType MTBDRplus version 2 line probe assay is equivalent to that of the Xpert MTB/RIF assay. J Clin Microbiol. 2012;50:3712-6. http://dx.doi.org/10.1128/JCM. 01958-12

20. Steingart KR, Sohn H, Schiller I, Kloda LA, Boehme CC, Pai M, et al. Xpert(R) MTB/RIF assay for pulmonary tuberculosis and rifampicin resistance in adults. Cochrane Database Syst Rev. 2013,1:CD009593. http://dx.doi.org/10. 1002/14651858.CD009593

21. Lemus $\mathbf{D}$. Métodos rápidos para la detección de resistencia en Mycobacterium tuberculosis (tesis). La Habana: Instituto de Medicina Tropical "Pedro Kouri”; 2007.

22. Golyshevskaia VI, Korneev AA, Chernousova LN, Selina LG, Kazarova TA, Grishina TD, et al. New microbiological techniques in diagnosis of tuberculosis. Probl Tuberk. 1996; 6:22-5.

23. Angeby KA, Klintz L, Hoffner SE. Rapid and inexpensive drug susceptibility testing of Mycobacterium tuberculosis with a nitrate reductase ass ay. J Clin Microbiol. 2002;40:553-5. http://dx.doi.org/10.1128/JCM.40.2.553-555.2002

24. Panaiotov S, Kantardjiev T. Nitrate reductase assay for drug susceptibility testing of Mycobacterium tuberculosis. J Clin Microbiol. 2002;40:3881-2. http://dx.doi.org/10.1128/ JCM.40.10.3881-3882.2002

25. Coban AY, Birinci A, Ekinci B, Durupinar B. Drug susceptibility testing of Mycobacterium tuberculosis with nitrate reductase assay. Int J Antimicrob Agents. 2004;24:304-6. http://dx.doi.org/10.1016/j.ijantimicag.2004.02.027
26. López M, Álvarez C, Imaz MS. Utility of nitrate reductase assay for detection of multidrug-resistant Mycobacterium tuberculosis in a low resource setting. Biomédica. 2011;31:258263. http://dx.doi.org/10.1590/S0120-41572011000200014

27. World Health Organization. Noncommercial culture and drug-susceptibility testing methods for screening patients at risk for multidrug-resistant tuberculosis: Policy statement. Geneva: World Health Organization; 2011.

28. Affolabi D, Odoun M, Sanoussi N, Martin A, Palomino JC, Kestens L, et al. Rapid and inexpensive detection of multidrug-resistant Mycobacterium tuberculosis with the nitrate reductase assay using liquid medium and direct application to sputum samples. J Clin Microbiol. 2008; 46:3243-5. http://dx.doi.org/10.1128/JCM.00083-08

29. Martin A, Palomino JC. Procedure manual nitrate reductase assay. Drug susceptibility testing for Mycobacterium tuberculosis. Antwerp, Belgium: Institute of Tropical Medicine, Mycobacteriology Unit; 2009.

30. Canetti G, Froman S, Grosset J, Hauduroy P, Langerova M, Mahler HT, et al. Mycobacteria: Laboratory methods for testing drug sensitivity and resistance. Bull World Health Organ. 1963;29:565-78.

31. Laszlo A, Rahman M, Espinal M, Raviglione $M$. Laboratories WINoSR. Quality assurance programme for drug susceptibility testing of Mycobacterium tuberculosis in the WHO/IUATLD Supranational Reference Laboratory Network: Five rounds of proficiency testing, 1994-1998. Int J Tuberc Lung Dis. 2002;6:748-56.

32. Ani AE, Daylop YB, Agbaji O, Idoko J. Drug susceptibility test of Mycobacterium tuberculosis by nitrate reductase assay. J Infect Dev Ctries. 2009;3:16-19.

33. Zabaleta-Vanegas AP, Llerena-Polo C, Orjuela-Gamboa DL, Valbuena-Arias YA, García-González LM, MejíaRestrepo G, et al. Evaluation of BACTEC MGIT 960 and the nitrate reductase assay in the National Laboratory Network of Colombia. Int J Tuberc Lung Dis. 2013;17:125-8. http:// dx.doi.org/10.5588/ijtld.11.0661

34. Shikama ML, Ferro e Silva R, Villela G, Sato DN, Martins MC, Giampaglia CM, et al. Multicentre study of nitrate reductase assay for rapid detection of rifampicin-resistant M. tuberculosis. Int J Tuberc Lung Dis. 2009;13:377-80.

35. Martin A, Montoro E, Lemus D, Simboli N, Morcillo N, Velasco M, et al. Multicenter evaluation of the nitrate reductase assay for drug resistance detection of Mycobacterium tuberculosis. J Microbiol Methods. 2005;63:145-50. http:// dx.doi.org/10.1016/j.mimet.2005.03.004

36. Gupta A, Sen MR, Mohapatra TM, Anupurba S. Evaluation of the performance of nitrate reductase assay for rapid drugsusceptibility testing of Mycobacterium tuberculosis in north India. J Health Popul Nutr. 2011;29:20-5.

37. Lemus D, Montoro E, Echemendia M, Martin A, Portaels F, Palomino JC. Nitrate reductase assay for detection of drug resistance in Mycobacterium tuberculosis: Simple and inexpensive method for low-resource laboratories. J Med Microbiol. 2006;55:861-3. http://dx.doi.org/10.1099/jmm.0. 46540-0

38. Kent PT KG. Public health mycobacteriology: A guide for the level III laboratory. Atlanta, GA: Centers for Disease Control. 1985. p. 96-103. 